\title{
Small bowel angioectasia as a marker of frailty and poor prognosis
}

\section{다 (1) $\odot$}

\author{
Authors \\ Alexander R. Robertson, Anastasios Koulaouzidis, William M. Brindle, Andrew J. Robertson, John N. Plevris
}

Institution

Department of Gastroenterology, Royal Infirmary of

Edinburgh, Edinburgh, Scotland

submitted 8.10 .2019

accepted after revision 12.2 .2020

Bibliography

DOI https://doi.org/10.1055/a-1180-8319 |

Endoscopy International Open 2020; 08: E953-E958

(c) Georg Thieme Verlag KG Stuttgart · New York

elSSN 2196-9736

Corresponding author

Dr. Alexander R. Robertson, MBChB MRCP (Lond) AFHEA,

Department of Gastroenterology, Royal Infirmary of

Edinburgh, 51 Little France Crescent, Edinburgh, EH16 4SA,

Scotland

Phone: + 01315361000

Alexander.Robertson@nhslothian.scot.nhs.uk

\section{ABSTRACT}

Background and study aims This study aimed to establish 5-year survival of patients diagnosed with bleeding small bowel (SB) angioectasia, with the hypothesis that many will suffer deaths relating to comorbidity rather than gastrointestinal bleeding.

Patients and methods SB capsule endoscopy (SBCE) procedures, performed for suspected SB bleeding or iron deficiency anemia, with angioectasia isolated as the cause of SB bleeding and at least 5 years of follow-up data were isolated $(n=125)$ along with an age-matched group with "normal" SBCE procedures $(n=125)$. These were retrospectively analysed with further information on mortality and comorbidity gathered through hospital records.

Results Those with angioectasia had a median age of 72.7 years and comorbidities were common. The 5 -year survival was $64.0 \%(80 / 125)$ compared to $70.4 \%(88 / 125)$ in those with "normal" SBCE. Those with significant cardiac or vascular comorbidity had a poorer survival $(52.9 \%$ (37/70) at 5 years) but anticoagulation/antiplatelets/ number of lesions or requirement endoscopic treatment seemed to make little difference. In those with SB bleeding secondary to angioectasia none of the subsequent deaths were directly attributable to gastrointestinal bleeding.

Conclusions In this cohort, SB angioectasia did not lead to any deaths but the 5 -year survival was poor due to those diagnosed often being older and having comorbidities. This would support the hypothesis that a diagnosis of SB bleeding secondary to angioectasia suggests frailty.

\section{Introduction}

Vascular lesions/angioectasia are the most common source of small bowel (SB) bleeding [1]. Although their pathogenesis is poorly understood, it is felt to relate to age and strain on the intestinal wall [2] with chronic hypoperfusion of the bowel hypothesized to contribute [3]. Mid gut bleeding is often precipitated by impaired clotting. This can be through medical anticoagulation and/or antiplatelet therapy or due to complicated and poorly understood sequalae of comorbidities, which often accumulate with age. For example, aortic valve stenosis (AoVS) is speculated to result in turbulent blood flow, which proteolyses von Willebrand (VWF) factor, and the resultant deficiency can propagate gastrointestinal bleeding [4]. Chronic kidney disease $(C K D)$ results in increased bleeding through impaired platelet function and platelet vessel wall interactions [5, 6]. As such, presentations with SB bleeding secondary to angioectasia could be felt to be a marker of frailty with age and comorbidity leading to initial formation and possible bleeding.

The aim of this study was to review survival in patients diagnosed with bleeding SB angioectasia, with the hypothesis that many will die due to organ failure and comorbidity rather than gastointestinal bleeding.

\section{Patients and methods}

A prospectively populated database of small bowel capsule endoscopy (SBCE) procedures was retrospectively reviewed for cases were angioectasia were isolated as the cause of SB bleeding. One hundred twenty-five SBCEs fulfilled the criteria (i.e. 
- Table 1 Comorbidities.

\begin{tabular}{|c|c|c|}
\hline \multirow[t]{2}{*}{ Comorbidity } & Angioectasia & Normal CE \\
\hline & Number (\%) & Number (\%) \\
\hline \multicolumn{3}{|l|}{ Cardiac/vascular } \\
\hline Ischemic heart disease & $28(22.4)$ & $25(20)$ \\
\hline LVSD/CCF & $24(19.2)$ & $17(13.6)$ \\
\hline Cerebrovascular disease & $19(15.2)$ & $15(12)$ \\
\hline Aortic valve disease $\pm \mathrm{AVR}$ & $18(14.4)$ & $10(8)$ \\
\hline Atrial fibrillation & $14(11.2)$ & $21(16.8)$ \\
\hline AAA & $11(8.8)$ & $1(0.8)$ \\
\hline Peripheral vascular disease & $11(8.8)$ & $4(3.2)$ \\
\hline Mitral valve disease & $3(2.4)$ & $8(6.4)$ \\
\hline Cardiomyopathy & $2(1.6)$ & $1(0.8)$ \\
\hline Mesenteric ischemia & $1(0.8)$ & 0 \\
\hline Diabetes & $30(24)$ & $23(18.4)$ \\
\hline \multicolumn{3}{|l|}{ Dementia } \\
\hline Vascular & $3(2.4)$ & $2(1.6)$ \\
\hline Alzheimer's & $2(1.6)$ & $3(2.4)$ \\
\hline Mixed & $1(0.8)$ & $2(1.6)$ \\
\hline Liver cirrhosis & $9(7.2)$ & $5(4.0)$ \\
\hline Viral hepatitis & 0 & $2(1.6)$ \\
\hline Crohn's disease & $4(3.2)$ & 0 \\
\hline Pancreatitis & 0 & $2(1.6)$ \\
\hline \multicolumn{3}{|l|}{ Renal } \\
\hline Chronic kidney disease & $18(14.4)$ & $9(7.2)$ \\
\hline End stage renal failure/dialysis & $4(3.2)$ & 0 \\
\hline \multicolumn{3}{|l|}{ Respiratory } \\
\hline COPD & $18(14.4)$ & $15(12)$ \\
\hline Asthma & $4(3.2)$ & $2(1.6)$ \\
\hline
\end{tabular}

angioectasia as the cause of SB bleeding and performed prior to June 2014, ensuring availability of 5-year survival data). They were retrospectively analysed with further information on the clinical course, management, number of angioectasia seen along with mortality and comorbidity gathered through hospital records.

Data from an age- and gender-matched group with "normal" SBCE procedures was also extracted. These patients were also all investigated for iron deficiency anemia or occult gastrointestinal bleeding but without any abnormality of the small bowel identified. Microsoft Excel (Microsoft Corporation, Washington, United States) was used to collate, analyse and graph the results.
- Table 1 (Continuation)

\begin{tabular}{|c|c|c|}
\hline \multirow[t]{2}{*}{ Comorbidity } & Angioectasia & Normal CE \\
\hline & Number (\%) & Number (\%) \\
\hline Bronchiectasis & 0 & $1(0.8)$ \\
\hline Pulmonary fibrosis & 0 & $2(1.6)$ \\
\hline $\begin{array}{l}\text { Hereditary hemorrhagic } \\
\text { telangectasia }\end{array}$ & $3(2.4)$ & 0 \\
\hline \multicolumn{3}{|l|}{ Hematological } \\
\hline Platelet release defect & $2(1.6)$ & 0 \\
\hline ITP & $1(0.8)$ & 0 \\
\hline Myelodysplasia & $1(0.8)$ & $1(0.8)$ \\
\hline Factor V Leiden & $1(0.8)$ & 0 \\
\hline VWF & $1(0.8)$ & $1(0.8)$ \\
\hline MGUS & 0 & $2(1.6)$ \\
\hline Borderline factor XI & 0 & $1(0.8)$ \\
\hline Vasculitis & 0 & $1(0.8)$ \\
\hline \multicolumn{3}{|l|}{ Malignancy } \\
\hline Breast & 0 & $4(3.2)$ \\
\hline Bladder & 0 & $1(0.8)$ \\
\hline Vulval & 0 & $1(0.8)$ \\
\hline Rectal & 0 & $1(0.8)$ \\
\hline Prostate & $1(0.8)$ & $2(1.6)$ \\
\hline Melanoma & 0 & $3(2.4)$ \\
\hline Parkinson's disease & 0 & $1(0.8)$ \\
\hline Hereditary spastic paraplegia & 0 & $1(0.8)$ \\
\hline \multicolumn{3}{|c|}{$\begin{array}{l}\text { CE, capsule endoscopy; LVSD, left ventricular systolic dysfunction; CCF, } \\
\text { congestive cardiac failure; AVR, aortic valve replacement; AAA, abdominal } \\
\text { aortic aneurysm; COPD, chronic obstructive pulmonary disease; ITP, im- } \\
\text { mune thrombocytopenic purpura; VWF, von Willebrand factor; MGUS, } \\
\text { monoclonal gammopathy of undetermined significance }\end{array}$} \\
\hline
\end{tabular}

\section{Results}

\section{Demographics and comorbidities}

At the time of SBCE, patients with isolated angioectasia had a median age of 72.7 (IQR 63.2-77.7) years, 69 female: 56 male. Those with a "normal" SBCE also had a median age of 72.7 (IQR 62.9-78.1) years, again 69 female: 56 male.

Comorbidities were common in both groups and are shown in $>$ Table 1. Cardiac, vascular, and renal disease was more common in those found to have angioectasia.

\section{Five-year survival}

Of the 125 patients with angioectasia on their SBCE, 64 had died by the end of the study period (June 2019) with 45 of these deaths within 5 years of their index SBCE ( $\downarrow$ Fig. 1$)$. Overall 


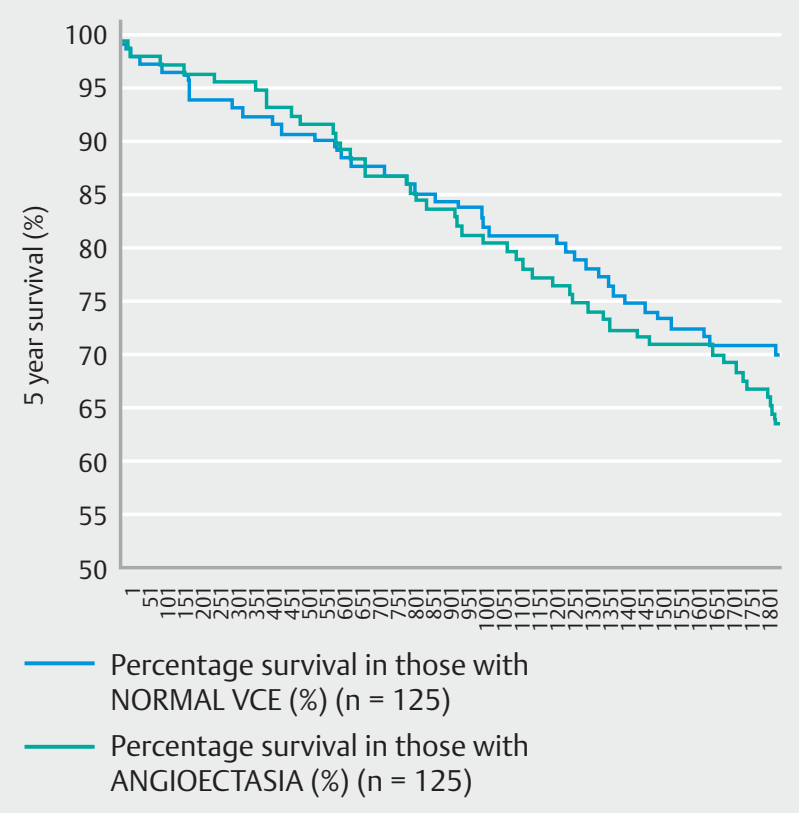

- Fig. 1 Five-year survival post diagnosis with angioectasia vs those with "normal" SBCE.

5 -year survival was $64.0 \%(80 / 125)$. Median age at death was 78 years (IQR 73-83) in those that died by the end of followup. Five-year survival was $70.4 \%$ (88/125 in those with "normal" SBCE. Median age at death in those who had died by the end of the study was 81.0 years.

\section{Impact of age and comorbidities}

Only $17.6 \%$ of patients (22/125) with angioectasia had no major comorbidities and they were younger with a median age of 69.3 (IQR 61.0-75.0) years. Eight of 22 had died by the end of follow-up (June 2019) with a median age of 78 years (IQR 75.5$78.25)$. The rate of 5 -year survival in those with no major comorbidities was $81.1 \%(18 / 22)$, as shown in > Fig. 2 .

Eighty-nine were over age 65 years with angioectasia, 12 had incomplete notes due to being out of area, and a further 11 had no major comorbidities (including those with uncomplicated Type 2 diabetes or hypertension only). This left 66 for analysis who were aged over 65 (mean age 76.4 years) with at least one major comorbidity. $28.8 \%$ (19/66) were alive at end of study period (June 2019) with a 5-year survival of $45.5 \%$ (30/66) (-Fig. 2).

In those with normal SBCE procedures with one or more major comorbidity and age $>65$, 5-year survival was $57.4 \%$ (39/68).

\section{Patients with renal, cardiac or vascular comorbidity}

Those with angioectasia and renal disease (CKD) had a 5-year survival of $61.9 \%(11 / 19)$.

Fifty-six percent (70/125) of those with angioectasia causing their SB blood loss had at least one major cardiac or vascular comorbidity (ischemic heart disease, congestive cardiac failure, cerebrovascular accident, aortic or mitral disease, atrial fibrilla-

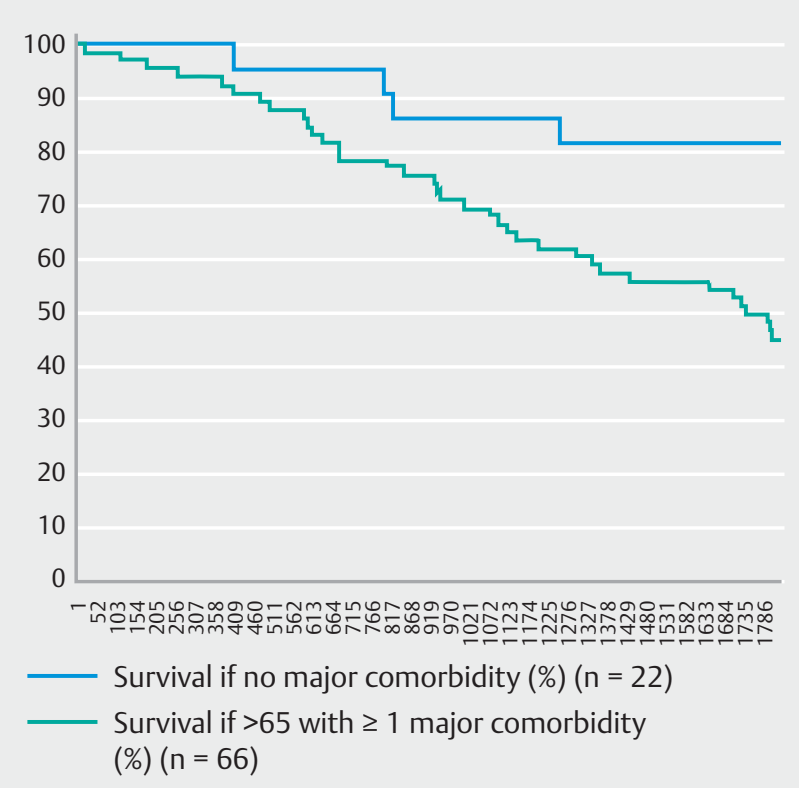

Fig. 2 Five-year survival in those with no major comorbidities compared to those $>65$ years of age with at least one major comorbidity following diagnosis of angioectasia .

tion, abdominal aortic aneurysm, peripheral vascular disease, cardiomyopathy, mesenteric ischemia, intracerebral bleed). Those with cardiac/vascular disease had a 5-year survival of $52.9 \%(37 / 70)$. They were slightly older at the time of SBCE with a median age of 75.5 (interquartile range 68.8-81.8) years. Those with cardiac/vascular disease taking antiplatelets/ anticoagulants had a 5 -year survival of $51.4 \%$, compared to $53.1 \%$ in those who were not (one excluded from analysis for unclear documentation).

Of those without angioectasia, 50.4\% (63/125) had cardiac or vascular comorbidity, and their 5-year survival rate was higher at $63.5 \%$.

\section{Causes of death}

None of the deaths in those with SB bleeding secondary to angioectasia were documented as being directly attributable to gastrointestinal bleeding, although a significant number were from out of area and had incomplete documentation available following their death ( $\triangleright$ Table 2 ).

Two deaths in the group with no SB abnormalities on CE were due to uncontrolled gastrointestinal bleeding, one case from rectal varices and one from a gastric ulcer. Both of these deaths occurred within a month of their normal SBCE investigation.

\section{Number and extent of angioectasia}

A clear description of the extent of small bowel angioectatic disease was present in 71 of the cases. Of them, 41/71 (57.7\%) had one or two angioectasias. The 5 -year survival in this group was $70.7 \%$ (29/41). This group had a median age of 70.0 (IQR 61.4-76.1) years, with 28 (68.3\%) suffering major cardiac, vascular or renal comorbidity. Sixteen $(39.0 \%)$ were noted to be 
- Table 2 List of causes of death.

\begin{tabular}{|c|c|c|}
\hline Pathology & $\begin{array}{l}\text { Deaths in } \\
\text { those with an- } \\
\text { gioectasia (\%) }\end{array}$ & $\begin{array}{l}\text { Deaths in those } \\
\text { without an- } \\
\text { gioectasia (\%) }\end{array}$ \\
\hline Pneumonia/chest sepsis & $7(5.6)$ & $6(4.8)$ \\
\hline MI & $5(4)$ & $5(4)$ \\
\hline Liver failure (NAFLD) & $2(1.6)$ & 0 \\
\hline COPD/bronchiectasis & $2(1.6)$ & $1(0.8)$ \\
\hline Cancer & $3(2.4)$ & $11(8.8)$ \\
\hline Bladder & $1(0.8)$ & 0 \\
\hline NSCLC & $1(0.8)$ & $3(2.4)$ \\
\hline SCLC & 0 & $2(1.6)$ \\
\hline $\mathrm{HCC}$ & 0 & $1(0.8)$ \\
\hline Breast & 0 & $1(0.8)$ \\
\hline Gastric & 0 & $1(0.8)$ \\
\hline Prostate & $1(0.8)$ & $2(1.6)$ \\
\hline Colon & 0 & $1(0.8)$ \\
\hline Following ascitic drain & 0 & $1(0.8)$ \\
\hline Surgical complication & $4(3.2)$ & 0 \\
\hline Renal & $1(0.8)$ & 0 \\
\hline $\begin{array}{l}\text { Crohn's resection with de- } \\
\text { hiscence of SB anastomosis }\end{array}$ & $1(0.8)$ & 0 \\
\hline $\begin{array}{l}\text { Adrenalectomy for Pheo- } \\
\text { chromocytoma }\end{array}$ & $1(0.8)$ & 0 \\
\hline Kidney/pancreas transplant & $1(0.8)$ & 0 \\
\hline Ruptured AAA & $2(1.6)$ & 0 \\
\hline CCF secondary to AS & $2(1.6)$ & $1(0.8)$ \\
\hline CVA & $2(1.6)$ & $2(1.6)$ \\
\hline CCF & $2(1.6)$ & $4(3.2)$ \\
\hline Renal failure & $2(1.6)$ & $1(0.8)$ \\
\hline Intracranial hemorrhage & $1(0.8)$ & $2(1.6)$ \\
\hline PE & $1(0.8)$ & 0 \\
\hline $\begin{array}{l}\text { Biliary sepsis secondary to } \\
\text { cholangiocarcinoma }\end{array}$ & $1(0.8)$ & 0 \\
\hline Ischemic colitis & $1(0.8)$ & $1(0.8)$ \\
\hline Rectal variceal bleed & 0 & $1(0.8)$ \\
\hline $\begin{array}{l}\text { Upper gastrointestinal } \\
\text { bleed (gastric ulcer) }\end{array}$ & 0 & $1(0.8)$ \\
\hline $\begin{array}{l}\text { Unclear/unrecorded (many } \\
\text { out of area) }\end{array}$ & $27(21.6)$ & $14(11.2)$ \\
\hline Alive & $61(48.8)$ & $74(59.2)$ \\
\hline \multicolumn{3}{|c|}{$\begin{array}{l}\text { MI, myocardial infarction; NAFLD, non-alcoholic fatty liver disease; COPD, } \\
\text { chronic obstructive pulmonary disease; NSCLC, non small cell lung carcino- } \\
\text { ma; HCC, hepatocellular carcinoma; SB, small bowel; AAA, abdominal aortic } \\
\text { aneurysm; CCF, congestive cardiac failure; AS, aortic stenosis; CVA, cere- } \\
\text { brovascular accident; PE, pulmonary embolism. }\end{array}$} \\
\hline
\end{tabular}

taking antiplatelets and/or anticoagulants. Thirty of 71 (42.3\%) of them had three or more angioectasias. This group was older with a median age of 76.2 years (IQR 69.3-80.4), with 19 $(63.3 \%)$ suffering major cardiac, vascular or renal comorbidity. Ten (33.3\%) were noted to be taking antiplatelets and/or anticoagulants. Their 5-year survival was $66.7 \%$ (20/30).

Those with documentation giving an unclear description of the number of lesions ( $n=54)$ were often older and largely from out of area. These patients had a median age of 71.8 and a 5 -year survival rate of $57.7 \%(31 / 54)$.

\section{Endoscopic treatments given}

Of the 125 patients with angioectasia isolated on their SBCE, 37 subsequently had endoscopic treatment with argon plasma coagulation (APC) planned (4 procedures later cancelled or failed): double balloon enteroscopy (DBE) $(n=22)$; push enteroscopy (PE) ( $n=5)$; upper gastrointestinal endoscopy $(n=9)$ and colonoscopy $(n=1)$. These patients had a median age of 74.1 years. Those who underwent endoscopic therapy had a 5-year survival rate of $62.2 \%(23 / 37)$.

\section{Discussion}

The overall 5-year survival in those diagnosed with angioectasia on SBCE was $64.0 \%$. This is comparable to the 5 -year mortality rate seen when SB adenocarcinoma (all SEER stages combined) is diagnosed (68\%) and worse than when the cancer is localised (85\% survival at 5 years) [7]. A similar study looking at all causes of occult SB bleeding had a $74.7 \%$ 5-year survival rate compared to $89.3 \%$ age matched cohort survival in Japan [8]. This would suggest that a diagnosis of SB angioectasia is both significant and associated with worse outcomes than serious alternative pathologies seen.

Those with a "normal" SB on SBCE (age and gender matched) were used as a comparison and had a 5-year survival rate of $70.4 \%$ ( $\triangleright$ Table 3 ). This is better than those with angioectasia but poorer than might have been expected. At ages 65 to 69 a man in the city of Edinburgh has a life expectancy of 18.0 years, and woman 20.5 years [9]. This could be explained in several ways. It seems likely that many of these patients will have had an anemia of chronic disease, which would be supported by the higher level of cancer deaths ( $8.8 \%$ vs $2.4 \%$ in those with angioectasia). It is also plausible that a significant number of those with a "normal" SBCE did in fact have iron deficiency or bleeding secondary to angioectasia either elsewhere within the gastrointestinal tract or missed within the small bowel. This could be suggested by a significant overlap in the comorbidities and clinical outcomes of the patients. It is possible some of these patients had pathology in their upper or lower gastrointestinal tract that had been missed on investigation. As such, the inclusion of those investigated for iron deficiency anemia or small bowel bleeding with a "normal" resultant CE is a poor control group and could not entirely be taken as such due to the degree of cohort bias. Ideally there would be an age matched healthy "control" group, but this is not available.

A reported $47 \%$ of elderly people in nursing homes are anemic [10], there is often only cursory investigation to exclude 
- Table 3 Summary of survival by subgroup.

\begin{tabular}{|l|l|c|c|}
\hline Group & Subgroup & Number & \% 5-year survival (n=) \\
\hline Angioectasia & No major comorbidities & 22 & $81.1 \%(18)$ \\
\hline Angioectasia & $1-2$ lesions & 41 & $70.7 \%(29)$ \\
\hline No angioectasia & All & 125 & $70.4 \%(88)$ \\
\hline Angioectasia & 3 of more lesions & 30 & $66.7 \%(20)$ \\
\hline Angioectasia & All & 125 & $64.0 \%(80)$ \\
\hline No angioectasia & Cardiac/vascular comorbidity & 63 & $63.5 \%(40)$ \\
\hline Angioectasia & Requiring endoscopic treatment & 37 & $62.2 \%(23)$ \\
\hline Angioectasia & Chronic kidney disease & 19 & $61.9 \%(11)$ \\
\hline No angioectasia & Over 65 with at least 1 major comorbidity & 68 & $57.4 \%(39)$ \\
\hline Angioectasia & Cardiac/vascular comorbidity without antiplatelets/anticoagulants & 32 & $53.1 \%(17)$ \\
\hline Angioectasia & All with cardiac/vascular comorbidity & 70 & $52.9 \%(37)$ \\
\hline Angioectasia & Cardiac/vascular comorbidity with antiplatelets/ anticoagulants & 37 & $51.4 \%(19)$ \\
\hline Angioectasia & Over 65 with at least 1 major comorbidity & 66 & $45.5 \%(19)$ \\
\hline
\end{tabular}

malignancy undertaken with symptomatic management following this. It is likely that a significant proportion of these patients will have angioectasia as the underlying pathology. Those undergoing SBCE are likely to represent a younger, healthier population, making the high mortality seen more striking and potentially over-estimating survival following a diagnosis of angioectasia. Despite this, those investigated in this study were older (median 72.7 years) and often multimorbid ( $\vee$ Table 1 ).

Although none of the deaths were directly caused by bleeding from angioectasia, it is not unlikely that chronic anaemia may have contributed to cardiovascular disease, or affected management options. Those who required endoscopic therapy following a diagnosis of angioectasia of the SB had almost the same survival as those who did not (62.2\% vs $64.0 \%)$. Those with one or two lesions were younger than those with three or more (70.0 vs 76.2 years) and had a marginally better 5-year survival rate $(70.1 \%$ vs $66.7 \%)$.

These results suggest that the degree of angioectatic bleeding was not associated with increased mortality but merely their presence could be taken as a marker of comorbidity and frailty.

This study has certain limitations. The retrospective design relied on data collected from prior to June 2014, with many of these patients from other health boards having incomplete records available. The original reports for many, as seen in the results section, was lacking with variable documentation of the lesions and clinical course at the time of these procedures.

The small numbers available are due to the specificity of the cohort being examined, but this does make result difficult to interpret. This is particularly true for the subgroups, therefore, generalization of these findings would be less reliable.

Characterization of the initial patient presentation and degree of angioectatic bleeding proved to be difficult. The focus of this manuscript is on the frailty of those presenting with an- gioectasia and this proved to complicate the severity markers investigated. The initial presentations, hemoglobin levels and transfusion requirements, where recorded, were less reliable and difficult to interpret as they were affected by confounding patient factors.

Despite these limitations, this study represents real-world outcomes in frail and elderly patients who are commonly excluded from prospectively held research. We feel this is a major strength of this manuscript.

Five-year survival was poorest in those over 65 with at least one major comorbidity (45.5\%). This is comparable with those who have metastatic small bowel adenocarcinoma diagnosed (42\% 5 year survival) [7].

\section{Conclusion}

As hypothesized, those diagnosed with SB angioectasia are very frequently elderly and frail, and although the angioectasia did not cause any documented deaths, the 5-year survival was poor.

\section{Competing interest}

The authors declare that they have no conflict of interest.

\section{References}

[1] Liao Z, Gao R, Xu C et al. Indications and detection, completion, and retention rates of small-bowel capsule endoscopy: a systematic review. Gastrointest Endosc 2010; 72: 280-286

[2] Warkentin TE, Moore JC, Anand SS et al. Gastrointestinal bleeding, angiodysplasia, cardiovascular disease, and acquired von Willebrand Syndrome. Transfus Med Rev 2003; 17: 272-286 
[3] Regula J, Wronska E, Pachlewski ]. Vascular lesions of the gastrointestinal tract. Best Pract Res Clin Gastroenterol 2008; 22: 313-328

[4] Sucker C, Michiels J], Zotz RB. Causes, etiology and diagnosis of acquired von willebrand disease: A prospective diagnostic workup to establish the most effective therapeutic strategies. Acta Haematol 2009; 121: 177-182

[5] Lutz J, Menke J, Sollinger D et al. Haemostasis in chronic kidney disease. Nephrol. Dial Transplant 2014; 29: 29-40

[6] Kaw D, Malhotra D. Platelet dysfunction and end-stage renal disease. Semin. Dial.19 Malden, USA: Blackwell Publishing Inc.; 2006: $317-$ 322
[7] Noone AM, Howlader N, Krapcho M et al. Cancer Statistics Review, 1975-2015 - SEER Statistics. SEER Cancer Stat. Rev; 2017

[8] Arai M, Matsumura T, Ohta Y et al. Long-term prognosis of patients with obscure gastrointestinal bleeding: a retrospective cohort study. Digestion 2019; 100: 37-44

[9] National Records of Scotland. Life Expectancy in Scottish Areas, 2015-2017. A National Statistics publication for Scotland. 2018

[10] Gaskell H, Derry S, Moore AR et al. Prevalence of anaemia in older persons: Systematic review. BMC Geriatr 2008; 8: 1 